\title{
Sun-glint imagery of Landsat 8 for ocean surface waves
}

\section{Ankita Misra, Bertrand Chapron, Frederic Nouguier, Balaji Ramakrishnan, Maria Yurovskaya}

Ankita Misra, Bertrand Chapron, Frederic Nouguier, Balaji Ramakrishnan, Maria Yurovskaya, "Sun-glint imagery of Landsat 8 for ocean surface waves," Proc. SPIE 10778, Remote Sensing of the Open and Coastal Ocean and Inland Waters, 107780L (24 October 2018); doi: 10.1117/12.2324754

Event: SPIE Asia-Pacific Remote Sensing, 2018, Honolulu, Hawaii, United States 


\title{
Sun glint Imagery of Landsat 8 for ocean surface waves
}

\author{
Ankita Misra $^{\mathrm{a}}$, Bertrand Chapron ${ }^{\mathrm{b}, \mathrm{c}}$, Frederic Nouguier ${ }^{\mathrm{b}}$, Balaji Ramakrishnan ${ }^{\mathrm{a}}$, Maria Yurovskaya ${ }^{\mathrm{c}, \mathrm{d}}$ \\ ${ }^{a}$ Indian Institute of Technology Bombay (India), Powai, Mumbai- India \\ ${ }^{\mathrm{b}}$ Ifremer (France), Centre De Brest Technopole De Brest -Iroise B.P. 70 29280, Plouzane, France \\ ${ }^{c}$ Russian State Hydrometeorologycal Institute, Saint Petersburg, Russia \\ ${ }^{\mathrm{d}}$ Marine Hydrophysical Institute of RAS, Sevastopol, Russia
}

\begin{abstract}
Local changes in specular reflections of visible sunlight on the ocean surfaces can be captured effectively by satellite sensors operating in the visible range of the electromagnetic spectrum. This causes the sun-glint imagery to closely resemble the oceanic images obtained using Synthetic Aperture Radar (SAR) further allowing the identification of the various fine scale structures and patterns of the ocean. Moreover, at relevant spatial resolutions, cloud-free conditions as well as optimum relative positions of the sensor, sun and the wave front it is possible to image ocean waves, wave transformations and refraction patterns using Satellite Sun-glint imagery (SSGI). In the present study, Landsat OLI imagery captured along the coast of Brest, France is used to derive ocean wave characteristics such as wavelength, direction, amplitude and then mapped to better understand the process of wave transformation. The 2D fast Fourier transform technique has been used on Band 5 (NIR, $0.851-0.879 \mu \mathrm{m})$ to derive the wavelength of swell waves in nearshore regions as well as to analyze the wavelength change. Furthermore, owing to the detector configuration of Landsat 8 OLI there is a small time lag between the channel acquisitions. This effectively helps to infer the space-time characteristics of the surface waves using the cross channel correlation between Band 5 and Band 6 subsequently enabling removal of the directional ambiguity associated with the wave spectra obtained from the analysis. The main purpose of this study is to demonstrate the importance of SSGI in deriving relevant coastal information which can be further utilized for bathymetry, surface current and wave motion determinations.
\end{abstract}

Keywords: Sun-glint Imagery, Wave transformation, Landsat 8 OLI, Fast Fourier Transform(FFT), cross-spectral analysis

\section{INTRODUCTION}

The reflectance over the oceans is significantly contributed by Sun-glint. The sea surface is smooth at the optical wavelength scale and acts as a near perfect specular reflector. The surface roughness dictates the intensity and pattern of the sunrays reflected upward close to the specular direction. The specular intensity and pattern is directly linked to the surface slope distribution and resultantly, optical methods have been used for measuring the slope probability distribution function (pdf), which can then be compared to the results of hydrodynamic modelling. Specular reflections can be widely observed in satellite imagery ${ }^{1}$. Especially operating in the visible range of the electromagnetic spectrum that can effectively capture fine contrast modulations related to local changes of the specular reflections of visible sunlight on the sensitive ocean surface. Satellite sun glint imagery (SSGI) contain significant information on the directional statistical properties of the wind ruffled sea surface roughness, especially its mean square slope (MSS), skewness, and kurtosis ${ }^{2,3}$.

Some of the earlier studies include the use of sun-glint imagery (astronaut photography, Landsat, Defense Meteorological Satellite Program (DMSP) satellites) for internal wave detection ${ }^{4,5,6,7}$. The differences in the internal wave signatures between sun-glint images from SPOT and Synthetic Aperture Radar was studied by Mitnik et al ${ }^{8}$. Jackson $^{9}$ discussed the characteristics of MODIS imagery and utilized true color sun-glint imagery for detection of highfrequency nonlinear internal waves. The internal solitary wave propagation in the South China Sea was mapped using 
Sun glint imagery ${ }^{10}$ and $250 \mathrm{~m}$ resolution MODIS SSGI images were used to report oil slicks by Hu et al ${ }^{11}$. A practical approach to convert sun glitter brightness variations into MSS anomalies was suggested by Kudryavtsev et al ${ }^{12,13}$ to quantify satellite observations of oil spills and sub-mesoscale ocean currents by Rascle et $\mathrm{a}^{14}$. The current research is motivated by the work done by Kudryavtsev et al. ${ }^{15}$ wherein they have suggested a method to quantitatively retrieve directional spectra of ocean surface waves from high-resolution satellite sun glitter imagery (SSGI) of Sentinel 2. In this study the objective is to use sun glint imagery of Landsat $8 \mathrm{OLI}$ to reveal and understand wave transformations. We further intend to use a combination of two bands to do a cross channel analysis to resolve the directional ambiguity of the waves. The overall goal is to obtain the required parameters for subsequently estimating bathymetry for near-shore regions.

\section{STUDY AREA}

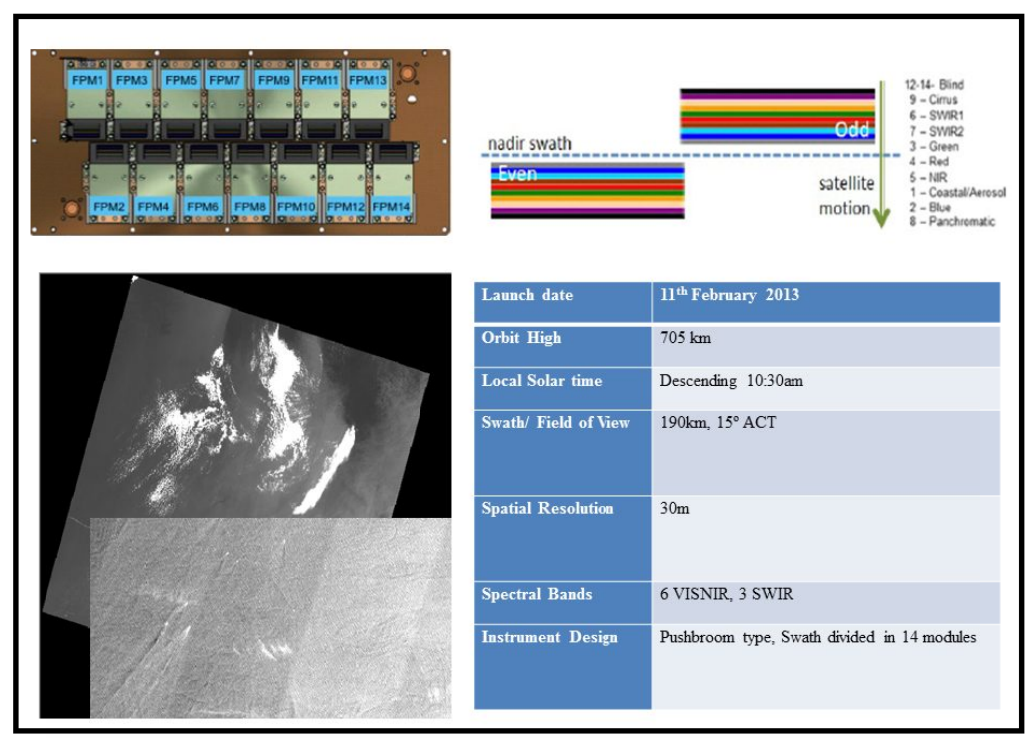

Figure 1 Configuration of Landsat 8 OLI data and relevance for Sun-glint Studies 


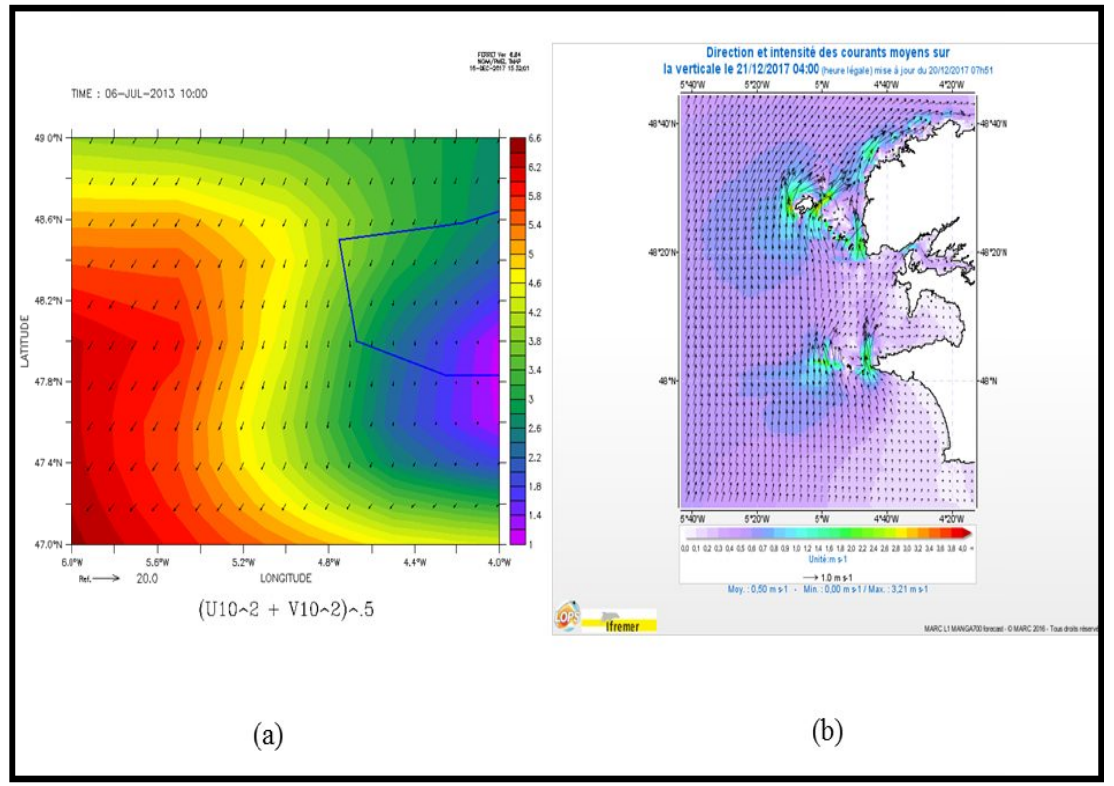

Figure 2. (a) Wind (b) Currents along the study area

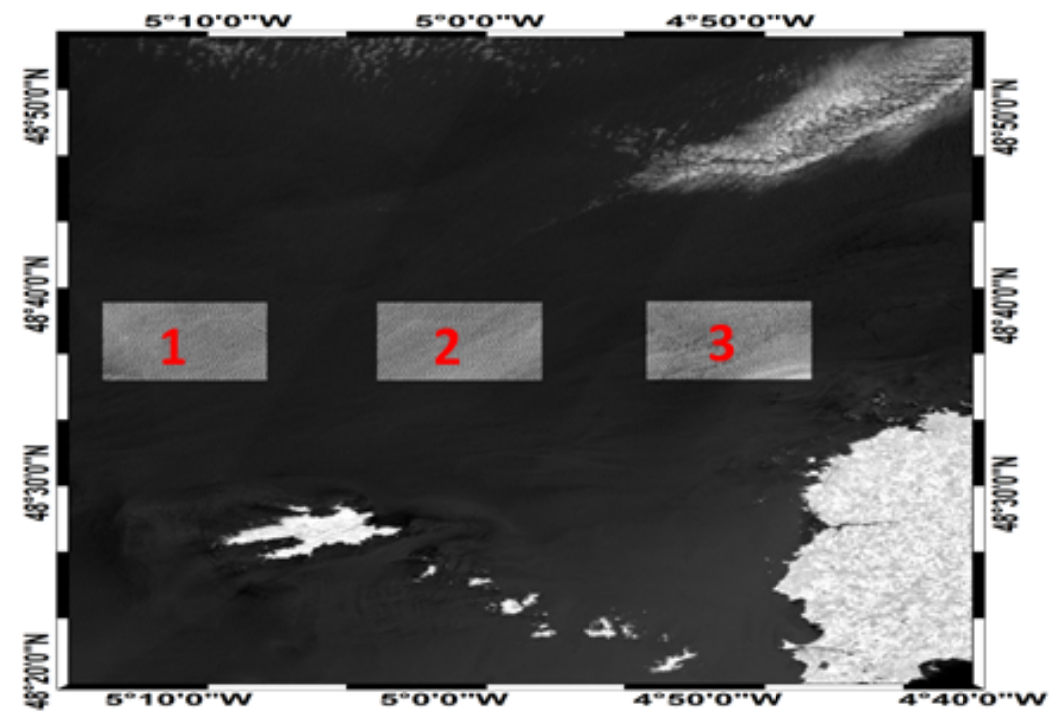

Figure 3. Study area- 3 subsets considered from offshore to near-shore

In this study, Landsat 8 OLI data of 6th July 2013 along the coast of Brest is utilized for analysis. Landsat 8 OLI data is a moderate resolution satellite imagery consisting of 11 spectral bands. The spatial resolutions of the bands 1-7 and 9 are $30 \mathrm{~m}, 15 \mathrm{~m}$ for band 8 , and $100 \mathrm{~m}$ for bands 10 and $11^{16}$. As can be seen in the Figure 1, the Landsat modules are arranged in even/odd order in a staggered row. Consequently, the even and odd modules view the earth with a light off nadir angle further resulting in a light parallax between the modules. Furthermore, since the spectral bands are placed successively in the along-track direction, there is also a parallax between the different spectral bands of a given module. This configuration of the Landsat 8 OLI can be further explored for sun glint based studies. This is because sun glint is a highly directional phenomena and a small change in the observation angle will lead to significant variations in the 
observed reflectances. The region considered has wind and current speeds ranging between $3.8-4.5 \mathrm{~m} / \mathrm{s}$ and $0.5-0.7 \mathrm{~m} / \mathrm{s}$ (Figure 2) respectively. For this analysis, initially, 3 subsets (Figure 3) are considered from offshore to near-shore where each subset is of the size 256 by 256 pixels, and each pixel is $30 \mathrm{~m}$. Further, the concept of cross spectral analysis is demonstrated and described using a single subset.

\section{METHODOLOGY}

\subsection{Condition for Sun-Glint Imagery}

In order to test for the condition for Sun-glint imagery the solar and sensor illumination angles of Landsat 8 OLI image is estimated. The surface brightness field in the sun glitter area where the impact of the sky radiance is negligible is considered here. Based on the Cox and $\mathrm{Munk}^{2}$, the sun glitter radiance, B generated by specular reflection of the sun glint is given by -

$$
B=\frac{\rho E_{S}}{4 \cos \theta_{v} \cos ^{4} \beta} P\left(Z_{1}, Z_{2}, S\right)
$$

Where,

$E_{\mathrm{s}}$ is the solar irradiance, $\rho$ is the Fresnal reflection coefficient, $\theta_{\mathrm{v}}$ is the view zenith angle, $\mathrm{P}$ is the 2D probability density function (PDF) of the sea slope $\mathrm{z}_{1}, \mathrm{z}_{2}$ in the two orthogonal directions in the $\mathrm{x}_{1}$ and $\mathrm{x}_{2}$ correspondingly, $\mathrm{S}$ is the generalized sea surface slope parameter.
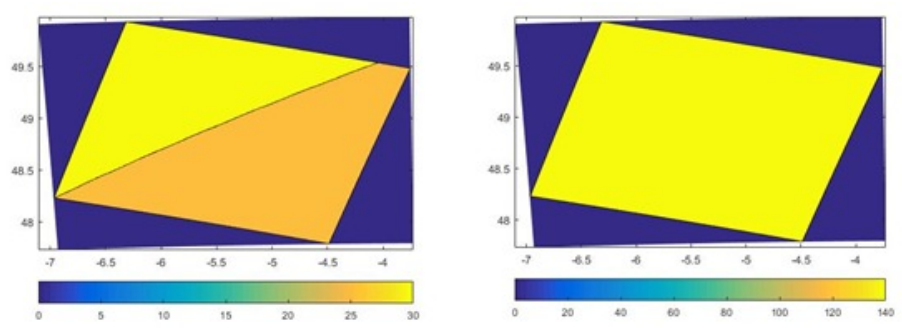

Solar Zenith

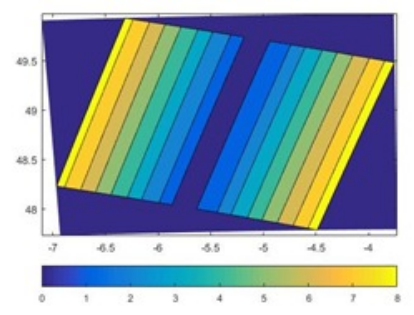

Sensor Zenith

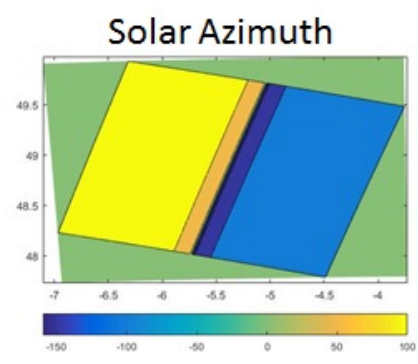

Sensor Azimuth

Figure 4. Solar and Sensor Zenith and Azimuth angles for the Landsat 8 OLI Imagery

The above equation states that $\mathrm{P}$ is the dependent on the statistical properties of the sea surface slopes, like mean square slope (MSS), skewness and peakedness, $Z_{1}$ and $Z_{2}$ denote the sea surface slopes that satisfy the conditions of specular reflections.

$$
\begin{aligned}
\mathrm{Z}_{1} & =\frac{\sin \theta_{\mathrm{s}} \cos \varphi_{\mathrm{s}}+\sin \theta_{\mathrm{v}} \cos \varphi_{\mathrm{v}}}{\cos \theta_{\mathrm{s}}+\cos \varphi_{\mathrm{v}}} \\
\mathrm{Z}_{2} & =\frac{\sin \theta_{\mathrm{s}} \sin \varphi_{\mathrm{s}}+\sin \theta_{\mathrm{v}} \sin \varphi_{\mathrm{V}}}{\cos \theta_{\mathrm{s}}+\cos \varphi_{\mathrm{v}}}
\end{aligned}
$$




$$
\tan \beta=\sqrt{z_{1}^{2}+z_{2}^{2}}
$$

Where,

$\theta_{S}$ is the sun zenith angle, $\theta_{v}$ is the sensor zenith angle, $\varphi_{S}$ sun azimuth angle and $\varphi_{S}$ is the sensor azimuth angle (Figure 4)

The PDF is further expressed in the normalized form as -

$$
P\left(Z_{1}, Z_{2}\right)=s^{-2} p\left(\frac{Z_{1}}{s}, \frac{Z_{2}}{s}\right)
$$

Where,

$\mathrm{s}^{2}$ is the mean squared slope (MSS) of sea surface, and $\mathrm{p}$ is a "scaled" PDF. The $\mathrm{s}^{2}$ is calculated following Cox and Munk (1954) for the given wind speed of the subset. The model is given as follows-

$$
\mathrm{s}^{2}=0.003+5.12 * 10^{3} * U_{w}
$$

Where,

$U_{w}$ is the wind speed $(\mathrm{m} / \mathrm{s})$.

The MSS is generally relevant in the case of wind waves shorter than $1 \mathrm{~m}$ and the shape of the sun glitter is dependent on the statistical properties of the short waves. For the present imagery, $\mathrm{Zn} / \mathrm{S} \sim 1$ and hence it satisfies the conditions of sun glint and can be used further for deriving ocean characteristics.

\subsection{Calculation of wavelength and phases of ocean waves}

In the presence of sun glint, the optical imagery strongly resembles an ocean synthetic aperture radar (SAR) image. Hence, 2D Fast Fourier Transform is applied to all the images to derive the dominant wavelength and phase for each sub-image.

The wave length and angle of propagation can be estimated as follows:

$$
\begin{aligned}
& \mathrm{L}=(\mathrm{N} \Delta \mathrm{x}) / \sqrt{\mathrm{u}^{2}+\mathrm{v}^{2}} \\
& \theta=\arctan \left(\frac{v}{u}\right)
\end{aligned}
$$

Where,

$\mathrm{L}$ is the measure peak wavelength, $\theta$ is the peak wave direction, $\Delta \mathrm{x}$ is the spatial resolution of the image, $\mathrm{N}$ is the size of the sub-image, $\mathrm{u}$ and $\mathrm{v}$ are coordinates of the dominant frequency with the center point as origin

\section{RESULTS AND DISCUSSION}

\section{Deriving dominant wavelength and phase changes using FFT}

The Landsat 8 OLI band 5 is used to apply the Fast Fourier Transform technique to derive the dominant Wavelength and Phase. Figure 5 shows the frequency domain obtained for each subset. Further it can be seen in table 1 that the wavelength shows a decreasing trend from offshore to shallow waters. The methodology clearly captures the wave transformation which could be attributed to bathymetry changes. However, this requires further investigation as this region is affected by very strong tidal currents which can also contribute to the observed dynamics in this region.

Nevertheless, considering the optical data follows the nature of a SAR imagery this concept can be further applied to derive the bathymetry of a region using the linear dispersion algorithm. 
Table 1. Wavelength and Phase Changes from Subset 1-3

\begin{tabular}{|l|r|r|}
\hline & L (meters) & $\Theta$ (degrees) \\
\hline Subset 1 & 164.41 & 28.23 \\
\hline Subset 2 & 163.32 & 16.11 \\
\hline Subset 3 & 157.57 & 32.37 \\
\hline
\end{tabular}

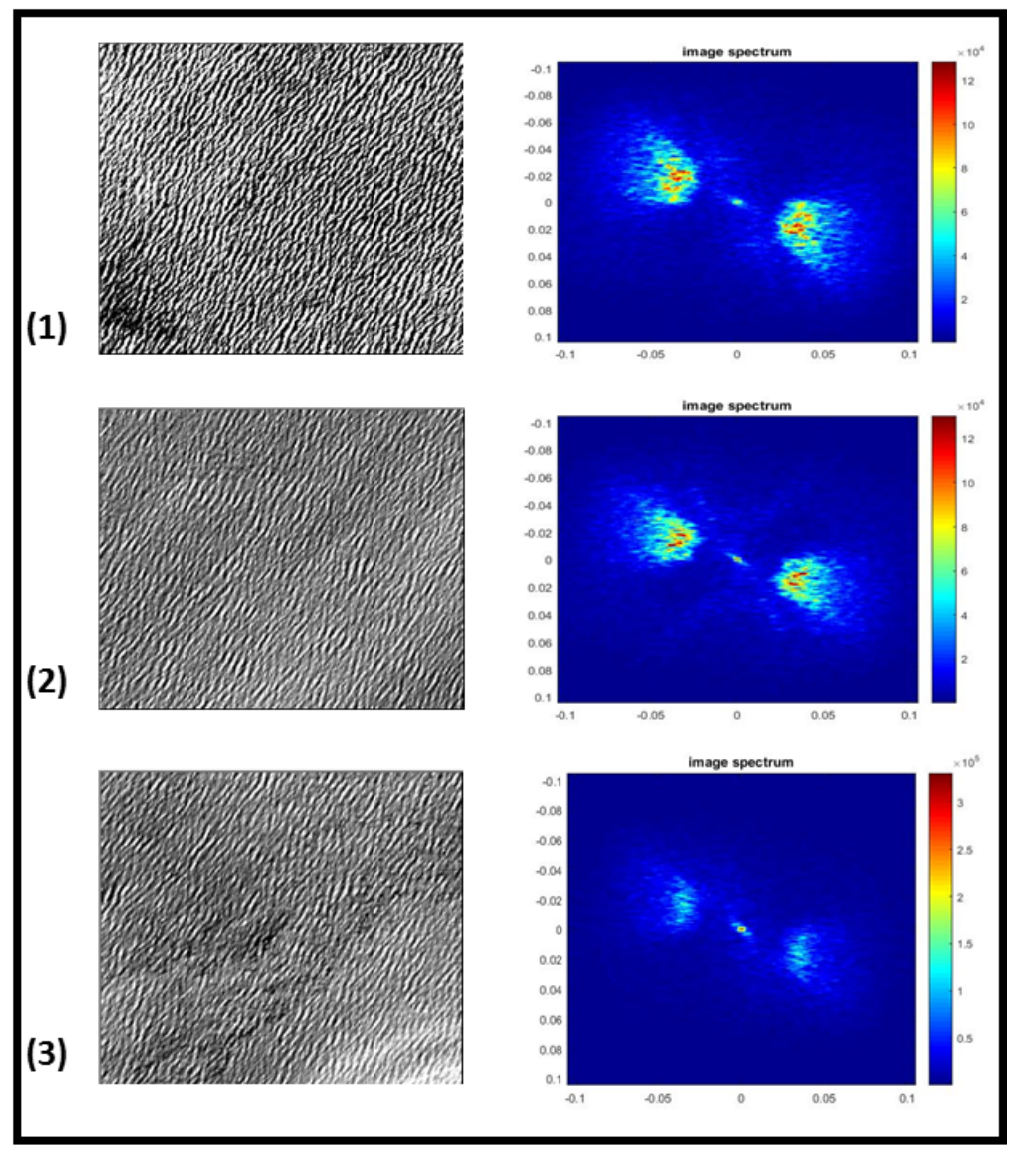

Figure 5. FFT results of the 3 subsets considered from offshore to near-shore

\section{Cross Spectral Analysis}

As mentioned earlier owing to the specific instrumentation and configuration, the Landsat 8 OLI image provides a scope to study the space -time characteristics of the detected surface waves. The bands chosen should be such that the cross channel parallax provides an optimal time lag which is long enough to estimate the phase velocity of the ocean waves and at the same time short enough to maintain a strong coherence between two consecutive observations. In order to select the best combination of bands that enable the optimum phase difference, the coherence and phase maps using the different band combinations were tested. Here, the application of the cross-spectral analysis will be demonstrated by using the subset 1 mentioned before. 
From figure 6 it can be seen band 5 and 6 satisfy the desirable condition. Therefore, based on the cross spectral analysis the phase shift $\varphi(\mathrm{k})$ derived for the given sub image is 30 degrees or 0.52 radians

This is further utilized for the calculation of $\omega$ further for the sub-image 1.

From Linear wave theory, the anticipated phase shift is -

$$
\varphi(\mathrm{k})=-\omega \Delta \mathrm{t}=-(\mathrm{gk})^{\frac{1}{2}} \Delta \mathrm{t}
$$

Where, $\Delta \mathrm{t}$ is the time delay between cross channel detectors which is calculated from the image azimuths for each channels.

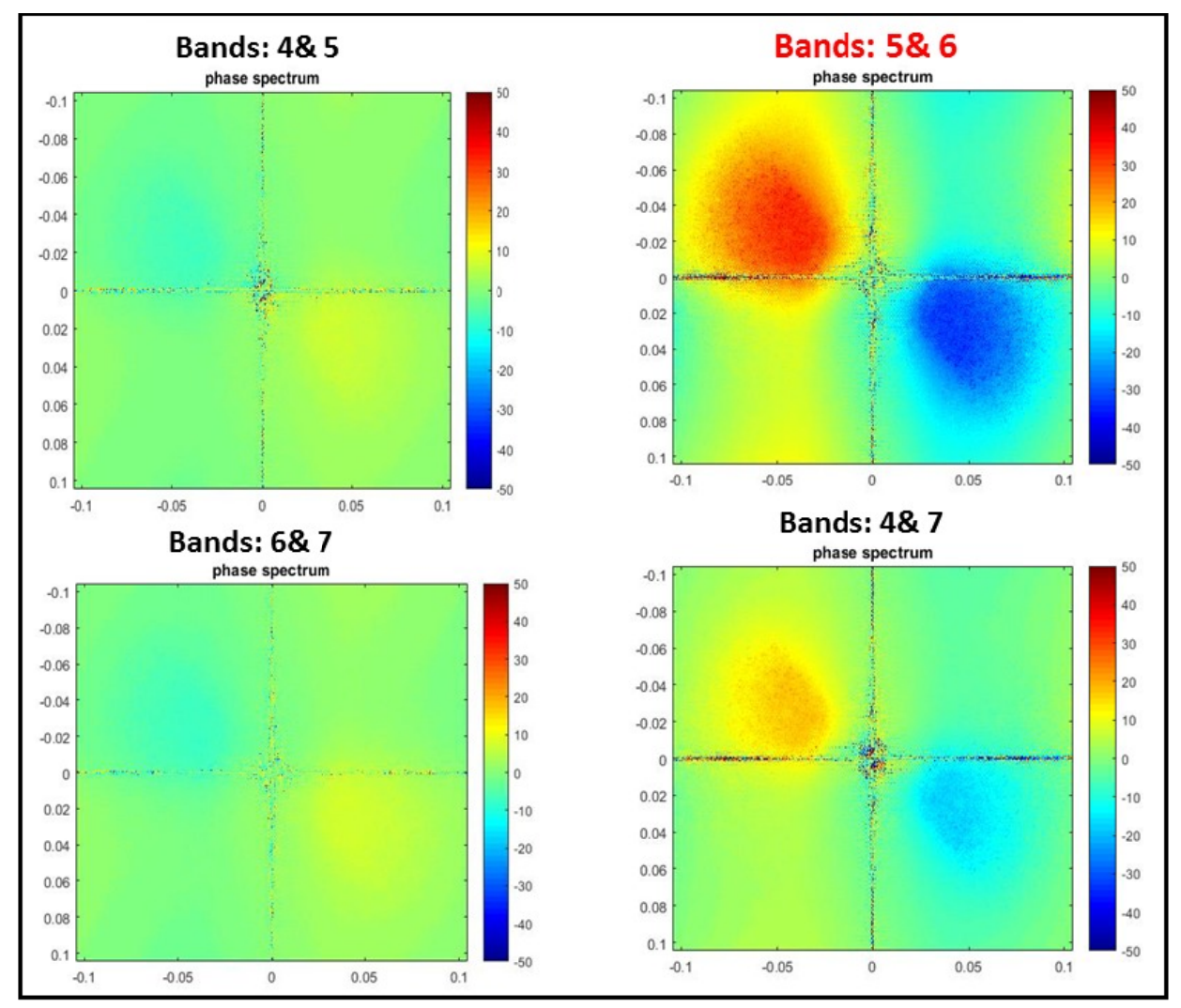

Figure 6. Cross-spectral analysis to find the optimal bands 


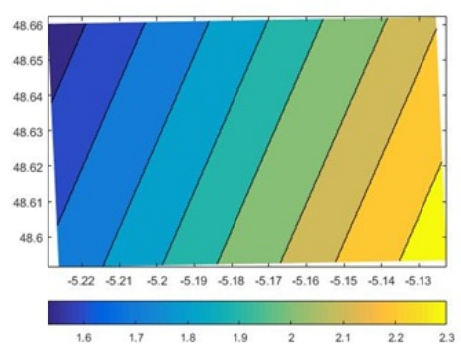

Sensor Zenith B05

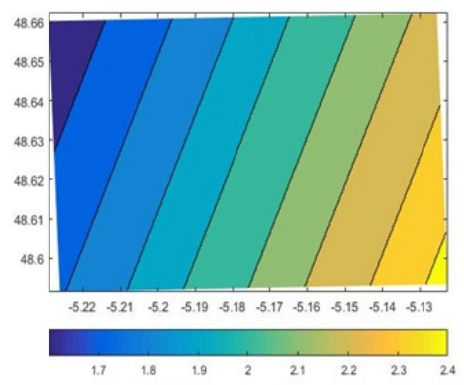

Sensor Zenith B06

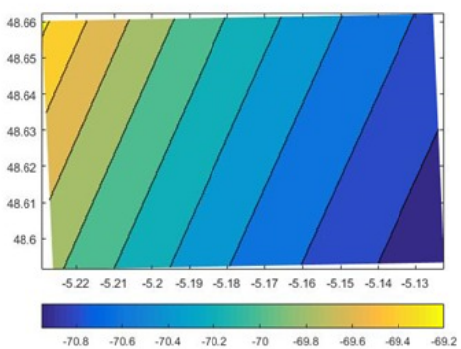

Sensor Azimuth B05

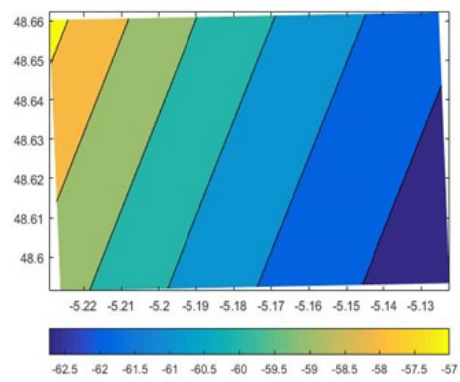

Sensor Azimuth B06

Figure 7. Sensor Zenith and Azimuth angles bot B05 and B06

The $\Delta \mathrm{t}$ is further calculated using the following equation as suggested by Kudryavtsev et al. ${ }^{15}$

$$
\begin{gathered}
\Delta \mathrm{t}=\frac{\mathrm{D}}{\mathrm{V}} \\
\mathrm{D}=\Delta \varphi_{\mathrm{v}} \mathrm{Htan} \theta_{\mathrm{v}} \\
V=\frac{V_{0} R_{E}}{\left(R_{E}+H\right)}
\end{gathered}
$$

Where,

$\mathrm{D}$ is the distance along the satellite trajectory, $\mathrm{H}$ is the satellite altitude, $\Delta \varphi_{v}$ is the azimuth difference between the bands, $\theta_{v}$ is the mean between sensor zenith angles between two channels, $\mathrm{V}$ is the ground speed estimated, $R_{E}$ is the radius of the earth and $V_{0}$ is satellite velocity

On calculation a $\Delta t$ value of $0.63 \mathrm{sec}$ and $\omega$ of 0.83 radians per seconds is obtained. This methodology can therefore be applied to the entire image using a moving window to obtain wave transformation maps of wavelengths and angular frequency and wave periods. However, certain limitations can be underlined in this analysis. Firstly, the resolution of Landsat 8 OLI image is $30 \mathrm{~m}$ which can prove to be relatively coarser to get the wave signatures. Secondly, the presence of currents in a particular region can result in errors in the estimations, especially on using this methodology for bathymetry estimation. This is specifically applicable in the case of the study region considered in this research.

\section{CONCLUSION}

In the present study we have demonstrated the use of Sun-glint Imagery of Landsat 8 OLI for obtaining wave transformation characteristics at the resolution of $30 \mathrm{~m}$. SSGI under optimal environmental conditions resembles SAR images which can be used to detect ocean surface waves, internal waves and fonts. Furthermore, the arrangement of the individual modules and bands of the Landsat sensor enables sufficient time lag to understand the space -time characteristics of the detected waves. Additionally, the wavelength and the true phase thus obtained can also be used to estimate the bathymetry near coastal regions where refraction and shoaling is observed. 


\section{ACKNOWLEDGMENT}

The work is supported by the Russian Science Foundation through the Project No.17-77-30019.

\section{REFERENCES}

[1] Khattak, S., Vaughan, R.A. and Cracknell, A.P., "Sunglint and its observation in AVHRR data," Remote Sensing of Environment 37(2),101-116 (1991).

[2] Cox, C. and Munk, W., "Measurement of the roughness of the sea surface from photographs of the sun's glitter, "Journal of the Optical Society of America 44(11), 838-850 (1954).

[3] Bréon, F.M. and Henriot, N., "Spaceborne observations of ocean glint reflectance and modeling of wave slope distributions," Journal of Geophysical Research: Oceans 111(C6)

[4] Apel, J. R., "Observations of internal wave surface signatures in ASTP photographs," Summary Sci. Rep. NASA SP-412, 505 - 509, Apollo-Soyuz Test Project, NASA Goddard Space Flight Cent., Greenbelt, Md. (1979)

[5] Apel, J.R., Byrne, H.M., Proni, J.R. and Charnell, R.L., "Observations of oceanic internal and surface waves from the Earth Resources Technology Satellite," Journal of Geophysical Research 80(6), 865-881(1975).

[6] Sawyer, C., "A satellite study of ocean internal waves," NOAA Tech. Memo. ERL PMEL, 46- 70, Pac. Mar. Environ. Lab., Seattle, Wash. (1983).

[7] Fett, R.W., La Violette, P.E., Nestor, M., Nickerson, J.W. and Rabe, K., "Navy Tactical Applications Guide," (2) Environmental Phenomena and Effects. BOHAN (WALTER A) CO PARK RIDGE IL. (1979).

[8] Mitnik, L., Alpers, W., Chen, K.S. and Chen, A.J., "Manifestation of internal solitary waves on ERS SAR and SPOT images: similarities and differences," In International geoscience and remote sensing symposium 5,18571859 (2000)

[9] Jackson, C., "Internal wave detection using the moderate resolution imaging spectroradiometer (MODIS)," Journal of Geophysical Research: Oceans 112(C11) (2007).

[10]Liu, B., Yang, H., Ding, X. and Li, X.," Tracking the internal waves in the South China Sea with environmental satellite sun glint images," Remote sensing letters 5(7), 609-618 (2014).

[11] Hu, C., Li, X., Pichel, W.G. and Muller-Karger, F.E., "Detection of natural oil slicks in the NW Gulf of Mexico using MODIS imagery" Geophysical Research Letters 36(1) (2009).

[12] Kudryavtsev, V., Myasoedov, A., Chapron, B., Johannessen, J. A., \& Collard, F., "Joint sun-glitter and radar imagery of surface slicks," Remote sensing of environment 120, 123-132 (2012).

[13] Kudryavtsev, V., Myasoedov, A., Chapron, B., Johannessen, J. A., \& Collard, F., "Imaging mesoscale upper ocean dynamics using synthetic aperture radar and optical data," Journal of Geophysical Research: Oceans, 117(C4).

[14] Rascle, N., Chapron, B., Ponte, A., Ardhuin, F. and Klein, P., "Surface roughness imaging of currents shows divergence and strain in the wind direction," Journal of Physical Oceanography 44(8), 2153-2163 (2014)

[15] Kudryavtsev, V., Yurovskaya, M., Chapron, B., Collard, F., \& Donlon, C., "Sun glitter imagery of ocean surface waves. Part 1: Directional spectrum retrieval and validation," Journal of Geophysical Research: Oceans 122(2), 1369-1383 (2017)

[16] L8-OLI mission description: http://landsat.usgs.gov/18handbook_section2.php 